\title{
Comprensión lectora digital vs. Tradicional según familiaridad con las TIC
}

\author{
Ana Alejandra Fuentes ${ }^{1}$, Virginia Jiménez ${ }^{2}$, y Jesús María Alvarado ${ }^{2}$ \\ ${ }^{1}$ Universidad Argentina de la Empresa (UADE) (Argentina); \\ ${ }^{2}$ Universidad Complutense de Madrid (España)
}

\begin{abstract}
Dado que existen diferencias en la literatura científica respecto a la lectura a través de dispositivos digitales, el presente trabajo tiene la finalidad de evaluar la comprensión lectora en función del manejo de las Tecnologías de la Información y la Comunicación (TIC), y la posibilidad de predecir el desempeño lector. Se evaluaron tres soportes: papel, ordenador y teléfono móvil o celular. Se trabajó con una muestra no probabilística e intencional de 178 estudiantes universitarios de la Universidad Complutense de Madrid. Se utilizaron tres lecturas seleccionadas ad-hoc para la investigación con sus respectivas preguntas y un cuestionario de usabilidad de las TIC. Los estudiantes leyeron los textos durante tres minutos, después respondieron 25 preguntas de opción múltiple por cada texto. En términos generales, el resultado de la investigación fue que la comprensión lectora mejora cuando se realiza en soporte ordenador. El estudio correlacional permite afirmar que la modalidad afecta diferencialmente a los sujetos. Así, dicho resultado está mediado por las habilidades, preferencias, usos y costumbres de los lectores. Se puede inferir que la sociedad de la información parece avanzar hacia una mejora en la comprensión lectora en medios no tradicionales de lectura respecto a los tradicionales.
\end{abstract}

Palabras clave: Comprensión lectora, TIC, predecir desempeño lector, ordenador, teléfono móvil.

Reading comprehension according to familiarity with ICT. Several differences were found in the scientific literature regarding reading through digital devices. The aim of the present work has been to study reading comprehension based onInformation and Communication Technologies (ICT) management and its possibility to predict reader performance.In order to achieve this, three supports were evaluated: paper, computer and mobile phone. We used a non-probabilistic and intentional sample of 178 university students from the Complutense University of Madrid. Three selected ad-hoc readings were used for the research with their respective questions and a questionnaire on ICT usability. Students read the texts for three minutes and then they answered a 25 multiple-choice questioner per text. In general terms, the result of the research showed that comprehension improves when students were tested on a computer. The correlation study allowed us to think that the modality affects differently the subjects. Thus, this result is mediated by the skills, preferences, uses and customs of the readers. So, it can be inferred that the information society seems to be moving towards an improvement in reading comprehension in non- traditional ways of reading compared to traditional ones.

Keywords: Reading comprehension, ICT, predict reading performance, computer, mobile phone.

Correspondencia Ana Alejandra Fuentes Cuiñas. Calle Lima, no 775. C.P.: 1073AAO. Ciudad Autónoma de Buenos Aires (Argentina). E-mail: afuentescuinas@uade.edu.ar 
Cómo la lectura en las pantallas difiere de la lectura en papel es relevante no solo para los más jóvenes, sino para casi todas las personas consideradas lectoras. Y son muchas las ventajas que ofrece el uso progresivo de libros digitales tanto en los espacios científicos como en los académicos, como por ejemplo el acceso inmediato, facilidad de mantenimiento y actualización, bajos costos, etc. (Romero-Otero, Iglesias-Fernández, y Giménez-Toledo, 2013). Si bien son varias las ventajas, sin embargo, un tema abierto y que ha generado controversia es el impacto sobre la comprensión lectora (Aatonen, Mannonen, Nieminen, y Nieminen, 2011; Liu, 2006; Wang y Lee, 2014; Yen y Wang, 2002).

Algunos estudios no han encontrado diferencias entre el entorno papel vs. digital, y otros han planteado que el entorno digital tiene efectos positivos (Salmerón y García, 2012). Por ejemplo, Bus y Neuman (2009) han encontrado que las aplicaciones multimedia pueden mejorar significativamente el desarrollo de la alfabetización en los estudiantes. Por otro lado, Singer y Alexander (2016) han señalado inconsistencias con respecto a las preferencias por entornos digitales y la comprensión lectora, teniendo en cuenta la preferencia y el juicio de eficacia en el desempeño en tareas de lectura.

Pero más allá de estas consideraciones, está claro que leer desde los diferentes formatos digitales, especialmente desde ordenadores, propone nuevos desafíos a los lectores (Aatonen et al., 2011; Bus y Neuman, 2009). Esto es así porque el medio juega un rol influyente bajo ciertas condiciones de texto o tarea o para ciertos lectores (Singer y Alexander, 2017). Hay consenso sobre que la lectura en pantalla introduce una mayor carga cognitiva comparada con la lectura en papel (Wästlund, Reinikka, Norlander, y Archer, 2005). De acuerdo con Sidi, Shpigelman, Zalmano, y Ackerman (2017), el medio proporciona una señal contextual que conduce a un procesamiento más superficial en la pantalla, independientemente de la longitud del texto, particularmente cuando las características de la tarea indican que el procesamiento superficial es legítimo. Asimismo, la comprensión lectora es el resultado de la interacción entre el conocimiento previo del lector, los procesos y las estrategias utilizadas para coordinar, y la información textual empleada para cumplir con las demandas del texto (Jiménez, Jiménez, Alvarado, y Puente, 2012; Jiménez, Jiménez, Puente, y Alvarado, 2011).

Por otra parte, Ackerman y Goldsmith (2011) han concluido que, la autorregulación de la comprensión lectora descendía cuando los estudiantes leían a través de medios electrónicos. Los investigadores encontraron un rendimiento más pobre en los textos leídos por pantalla en comparación con el contexto tradicional en lo que respecta al esfuerzo, la regulación, el rendimiento de las pruebas y el grado de exceso de confianza (Sidi et al., 2017).

Puesto que existen numerosas controversias sobre el soporte más efectivo en relación con la comprensión de la lectura, esta investigación tiene un doble objetivo, evaluar si la comprensión lectora está influenciada por el soporte en el que se lee, y hasta 
qué punto la familiaridad con las TIC puede explicar el rendimiento en los distintos soportes.

\section{MÉTODO}

\section{Participantes}

Participaron en el estudio, de forma voluntaria, 178 estudiantes universitarios que cursaban $1^{\circ}$ de Grado de Trabajo Social en la Universidad Complutense de Madrid durante el año académico 2016-2017. El rango de edad de los participantes fue de 18 a 25 años, siendo el $75 \%$ mujeres y el $25 \%$ hombres.

\section{Instrumentos}

Tres textos seleccionados ad-hoc para la investigación de longitud similar (363, 383 y 379 palabras) de tres libros: Ensayo sobre la ceguera (Texto 1), La balsa de piedra (Texto 2) y El hombre duplicado (Texto 3), del escritor José Saramago. La necesidad de utilizar tres textos se justifica por el diseño de medidas repetidas, y la selección de textos de un mismo autor se realizó para evitar diferencias en comprensión debidas al material de evaluación. Las 25 preguntas diseñadas para evaluar la comprensión de los textos mostraron las siguientes fiabilidades: Texto 1 con un alfa de $\mathrm{Cronbach}=.713$, el Texto 2 con un alfa de Cronbach $=.834$ y Texto 3 con un alfa de Cronbach $=.845$.

CUTIC: Cuestionario sobre usabilidad de dispositivos con conexión a Internet (Jiménez, Alvarado, y Llopis, 2017). CUTIC ha sido diseñado para medir aspectos relacionados con el uso de las Tecnologías de la Información y la Comunicación (TIC). Recoge datos de conductas digitales y opiniones sobre la utilidad de las TIC en el ámbito educativo, así como emociones generadas ante el uso de ellas, en dos soportes: ordenador o tableta y teléfono móvil (celular). Consta de 46 ítems distribuidos en dos grupos de 23 ítems. Los primeros corresponden al soporte ordenador o tableta y los segundos corresponden al soporte del teléfono móvil (celular). Los ítems de ambos grupos son idénticos. La diferencia está en el soporte al que van dirigidos.

\section{Procedimiento}

Los participantes de la muestra se asignaron aleatoriamente a 9 grupos en forma aleatoria a cada condición experimental (impresa, digital en teléfono móvil y digital en ordenador). Todos los estudiantes que formaron parte de la muestra resolvieron las preguntas de los textos en las tres condiciones experimentales.

Durante la primera semana del estudio los estudiantes contestaron el CUTIC (Jiménez et al., 2017). La siguiente semana los estudiantes leyeron los textos en las tres condiciones de lectura: en papel (o lectura tradicional), en teléfono móvil (celular) y en ordenador. A continuación de la lectura de cada texto los estudiantes contestaron 25 
preguntas de comprensión sobre dichos textos. Todos los estudiantes que participaron lo hicieron en forma voluntaria, sin recibir compensación.

Para el análisis de datos se utilizó el programa estadístico SPSS versión 22.

\section{RESULTADOS}

Para evaluar las posibles diferencias entre soportes se realizó un ANOVA de medidas repetidas que reveló un efecto principal significativo del factor Soporte $F(2,314)=17,068, p<0.01, \eta^{2}$ parcial $=0.10$. Los resultados de la evaluación fueron mejores en la presentación en el soporte ordenador $(M=16.51, S D=3.99)$ seguido de la presentación en papel $(M=15.33, S D=4.14)$ y en último lugar la presentación mediante teléfono móvil $(M=14.02, S D=4.27)$. El análisis post hoc Bonferroni, indicó que todas las diferencias eran estadísticamente significativas a $p<0.05$.

Por otra parte, las correlaciones entre las puntuaciones de los participantes en los distintos soportes fueron bajas o no significativas: papel-ordenador $r=.11(p=0.16)$, papel-teléfono móvil $r=.16$ ( $p=0.37)$ y teléfono móvil- ordenador $r=.17(p=0.26)$. En consecuencia, la modalidad afecta diferencialmente a la comprensión lectora de los sujetos, siendo una posible explicación el uso de las tecnologías. Para testar esta hipótesis se realizaron análisis de regresión logística. Para ello, fue preciso dicotomizar las variables en cero y uno en función de los resultados en los distintos soportes. Se introdujeron como variables predictoras los ítems del cuestionario CUTIC que fueron sensibles a este análisis (ver tabla 1).

Tabla 1. Distribución de Ítems en Función del Soporte y las Variables Comprometidas

\begin{tabular}{lccc}
\hline & \multicolumn{1}{c}{ Descripción } & $\begin{array}{c}\text { Soporte } \\
\text { Ordenador/tableta }\end{array}$ & $\begin{array}{c}\text { Soporte } \\
\text { Teléfono móvil }\end{array}$ \\
\hline Variables & $\begin{array}{c}\text { Iiempo de uso de internet, de } \\
\text { mensajería y de juegos, así como en } \\
\text { el estudio y en las RRSS }\end{array}$ & $2,5,9,21$ & $24,27,28,33$ \\
\hline $\begin{array}{l}\text { Frecuencia, uso y ámbito } \\
\text { Frecuencia y Redes }\end{array}$ & $\begin{array}{c}\text { Tiempo de uso de Facebook, } \\
\text { Twitter, Instagram, Youtube, otras }\end{array}$ & 12 & $35,36,37,38,39$ \\
\hline Cociales (RRSS) & Relajación, adicción & 18,19 & 41,42 \\
\hline
\end{tabular}

En la regresión logística para papel vs ordenador, se obtuvo un modelo que permitía hacer un $67.4 \%$ de clasificaciones correctas $(60.2 \%$ papel y $73.7 \%$ ordenador), siendo las variables que permiten hacer esta predicción las que se indican en la tabla 2. 
Tabla 2. Ítems que Permiten Discriminar Comprensión Lectora en Formatos Papel y Ordenador

\begin{tabular}{lccccc}
\hline & B & Error estándar & Wald & Sig. & Exp(B) \\
\hline $\begin{array}{l}\text { Item } 5 \text { ¿Con qué frecuencia al día juegas por Internet en } \\
\text { ordenador y/o tableta? }\end{array}$ & .713 & .294 & 5.875 & .015 & .490 \\
$\begin{array}{l}\text { Item } 9 \text { ¿Con qué frecuencia al día utilizas internet desde } \\
\text { dispositivos como ordenador o tableta en los estudios? }\end{array}$ & .336 & .177 & 3.577 & .059 & .715 \\
\hline $\begin{array}{l}\text { İem 18. He dejado de hacer alguna actividad (ir al cine, } \\
\text { salir con amigos, ..) por estar conectado/a a Internet con } \\
\text { el ordenador y/o tableta }\end{array}$ & .936 & .370 & 6.422 & .011 & 2.551 \\
$\begin{array}{l}\text { İtem 19. Encuentro tranquilidad, alivio, desahogo cuando } \\
\text { navego por la Red con el ordenador y/o tableta }\end{array}$ & .726 & .215 & 11.366 & .001 & .484 \\
$\begin{array}{l}\text { İem 21 ¿Consideras que es útil el uso de Internet a través } \\
\text { del ordenador y/o la tableta en el ámbito educativo en el } \\
\text { trabajo en grupo? }\end{array}$ & -.332 & .167 & 3.976 & .046 & 1.394 \\
\hline $\begin{array}{l}\text { Item 36 ¿Con qué frecuencia al día utilizas Twitter desde } \\
\text { el teléfono móvil (celular) }\end{array}$ & -.453 & .191 & 5.614 & .018 & 1.572 \\
\hline $\begin{array}{l}\text { Item 38 ¿Con qué frecuencia al día utilizas Youtube desde } \\
\text { el teléfono móvil (celular)? }\end{array}$ & -.380 & .199 & 3.637 & .057 & 1.462 \\
\hline $\begin{array}{l}\text { Item 39 ¿Con qué frecuencia al día utilizas otras redes } \\
\text { desde el teléfono móvil (celular)? }\end{array}$ & .352 & .188 & 3.502 & .061 & .703 \\
\hline Constante & .069 & .462 & .022 & .882 & .934 \\
\hline
\end{tabular}

En la regresión logística para papel vs teléfono móvil, se obtuvo un modelo que permitía hacer un $66.9 \%$ de clasificaciones correctas (69.3\% papel y $64.4 \%$ ordenador), siendo las variables que permiten hace esta predicción las que se indican en la tabla 3.

Tabla 3. Ítems que Permiten Discriminar Comprensión Lectora en Formatos Papel y Teléfono Móvil

\begin{tabular}{|c|c|c|c|c|c|}
\hline & B & $\begin{array}{c}\text { Error } \\
\text { estándar }\end{array}$ & Wald & Sig. & $\operatorname{Exp}(B)$ \\
\hline $\begin{array}{l}\text { Ítem } 2 \text { ¿Durante cuánto tiempo usas internet al } \\
\text { día en el ordenador o la tableta? }\end{array}$ & -.353 & .174 & $\begin{array}{c}4.13 \\
0\end{array}$ & $\begin{array}{c}.04 \\
2 \\
\end{array}$ & .702 \\
\hline $\begin{array}{l}\text { Ítem } 5 \text { ¿Con qué frecuencia al día juegas por } \\
\text { Internet en ordenador y/o tableta? }\end{array}$ & .723 & .273 & $\begin{array}{c}6.98 \\
4\end{array}$ & $\begin{array}{c}.00 \\
8\end{array}$ & 2.060 \\
\hline $\begin{array}{l}\text { Ítem } 9 \text { ¿Con qué frecuencia al día utilizas } \\
\text { internet desde dispositivos como ordenador o } \\
\text { tableta en los estudios? }\end{array}$ & .463 & .187 & $\begin{array}{c}6.16 \\
6\end{array}$ & $\begin{array}{c}.01 \\
3\end{array}$ & 1.589 \\
\hline $\begin{array}{l}\text { Ítem } 12 \text { ¿Con qué frecuencia al día utilizas } \\
\text { Facebook desde soportes como ordenador y/o } \\
\text { tableta? }\end{array}$ & -.534 & .222 & $\begin{array}{c}5.79 \\
0\end{array}$ & $\begin{array}{c}.01 \\
6\end{array}$ & .586 \\
\hline $\begin{array}{l}\text { Ítem } 21 \text { ¿Consideras que es útil el uso de } \\
\text { Internet a través del ordenador y/o la tableta } \\
\text { en el ámbito educativo en el trabajo en grupo? }\end{array}$ & -.431 & .174 & $\begin{array}{c}6.13 \\
1\end{array}$ & $\begin{array}{c}.01 \\
3\end{array}$ & .650 \\
\hline $\begin{array}{l}\text { Ítem } 35 \text { ¿Con qué frecuencia al día utilizas } \\
\text { Facebook desde el teléfono móvil (celular)? }\end{array}$ & .341 & .190 & $\begin{array}{c}3.20 \\
5\end{array}$ & $\begin{array}{c}.07 \\
3\end{array}$ & 1.406 \\
\hline $\begin{array}{l}\text { Ítem } 38 \text { ¿Con qué frecuencia al día utilizas } \\
\text { Youtube desde el teléfono móvil (celular)? }\end{array}$ & -.689 & .207 & $\begin{array}{c}11.03 \\
2\end{array}$ & $\begin{array}{c}.00 \\
1\end{array}$ & .502 \\
\hline $\begin{array}{l}\text { Ítem } 39 \text { ¿Con qué frecuencia al día utilizas otras } \\
\text { redes desde el teléfono móvil (celular)? }\end{array}$ & .403 & .180 & $\begin{array}{c}5.02 \\
5\end{array}$ & $\begin{array}{c}.02 \\
5\end{array}$ & 1.496 \\
\hline $\begin{array}{l}\text { Ítem 42. Encuentro tranquilidad, alivio, } \\
\text { desahogo cuando navego por la Red con el } \\
\text { teléfono móvil (celular) }\end{array}$ & 513 & 202 & .470 & 011 & .671 \\
\hline Constante & 534 & 490 & .188 & 276 & .706 \\
\hline
\end{tabular}


Tabla 4. Ítems que Permiten Discriminar Comprensión Lectora en Formatos Ordenador y Teléfono Móvil

\begin{tabular}{|c|c|c|c|c|c|}
\hline & $\mathrm{B}$ & $\begin{array}{c}\text { Error } \\
\text { estándar }\end{array}$ & Wald & Sig. & $\operatorname{Exp}(B)$ \\
\hline $\begin{array}{l}\text { Ítem } 12 \text { ¿Con qué frecuencia al día utilizas Facebook desde } \\
\text { soportes como ordenador y/o tableta? }\end{array}$ & .574 & .204 & 7.935 & .005 & .563 \\
\hline $\begin{array}{l}\text { Ítem } 24 \text { ¿Cuánto tiempo hace que usas internet desde el } \\
\text { teléfono móvil } \\
\text { (celular)? }\end{array}$ & .377 & .191 & 3.920 & .048 & .686 \\
\hline $\begin{array}{l}\text { Ítem } 27 \text { ¿Con qué frecuencia al día utilizas servicios de } \\
\text { mensajería } \\
\text { (WhatsApp, chat) en el teléfono móvil (celular)? }\end{array}$ & 617 & .185 & 11.139 & .001 & 1.853 \\
\hline $\begin{array}{l}\text { İtem } 28 \text { ¿Con qué frecuencia al día juegas por Internet en el } \\
\text { teléfono móvil (celular)? }\end{array}$ & 522 & .289 & 3.262 & .071 & 1.686 \\
\hline $\begin{array}{l}\text { Ítem } 33 \text { ¿Con qué frecuencia al día te conectas a Internet } \\
\text { desde el teléfono móvil (celular) para usar las Redes } \\
\text { Sociales? }\end{array}$ & .348 & .190 & 3.372 & .066 & .706 \\
\hline $\begin{array}{l}\text { Ítem } 37 \text { ¿Con qué frecuencia al día utilizas Instagram desde el } \\
\text { teléfono } \\
\text { móvil (celular)? }\end{array}$ & .393 & .191 & 4.239 & .039 & .675 \\
\hline $\begin{array}{l}\text { İtem } 41 . \text { He dejado de hacer alguna actividad (ir al cine, salir } \\
\text { con amigos, } \\
\text {..) por estar conectado/a a Internet con el teléfono móvil } \\
\text { (celular) }\end{array}$ & 951 & .463 & 4.231 & .040 & 2.589 \\
\hline Constante & .725 & .675 & 6.528 & .011 & 5.614 \\
\hline
\end{tabular}

Finalmente, en la regresión logística para ordenador vs teléfono móvil, se obtuvo un modelo que permitía hacer un $73.0 \%$ de clasificaciones correctas $(93.2 \%$ ordenador y $33.3 \%$ móvil), siendo las variables que permiten hace esta predicción las que se indican en la tabla 4.

\section{CONCLUSIONES/DISCUSIÓN}

Los ítems englobados en la variable "Frecuencia, Uso y Ámbito" referida al tiempo de uso de Internet, de mensajería y de juegos, así como la frecuencia y uso en el estudio y en las RRSS (ver tabla 1) reflejan, como era de esperar, que cuanto más uso y frecuencia los sujetos tengan de estos dispositivos, más destreza tendrán en competencia digital. Parece, además, que los estudiantes que conforman la muestra utilizan el ordenador o la tableta para el estudio más que el teléfono móvil o celular y éste más para conectarse a las RRSS. El ordenador es un soporte que favorece más su uso para la elaboración de trabajos demandados en la universidad y/o estudio que el teléfono móvil. Puede que esto sea debido al tamaño de la pantalla y del teclado; ya que la consulta y elaboración de cualquier documento es más cómoda en soporte ordenador o tableta. Parece que la utilidad percibida es para el trabajo en grupo. Sin embargo, las RRSS son accesibles cómodamente desde cualquier tipo de dispositivo y los sujetos que conforman la muestra tienen acceso a un teléfono móvil o celular en cualquier momento y lugar (ubicuidad de la tecnología) por lo que la conexión a Internet para el acceso a las RRSS es más inmediata desde este dispositivo. Esto se reafirma con los ítems englobados en la variable "Frecuencia y Redes 
Sociales" que tienen que ver con la frecuencia de uso de las RRSS más utilizadas actualmente.

Los ítems englobados en la variable "Conducta/emoción" son los referidos a la conducta de adicción y emociones provocadas por el acceso o no a las TIC con conexión a Internet. El estudio realizado por De Sola, Talledo, Rubio, y Rodríguez (2017) observa que alrededor del 15,4\% de la población española utiliza el teléfono móvil mucho tiempo a lo largo del día interfiriendo en actividades cotidianas. Esta población se encuentra en riesgo de padecer mayores complicaciones. Este mismo estudio constata que el 5,1\% de la población ya se encuentra sumido en una adicción.

Actualmente, el uso de la tecnología implica necesariamente la tarea de leer: mensajes, historias, noticias..., cualquier evento notificable por escrito tiene como objetivo el ser leído; por lo que la destreza lectora para la comprensión de los distintos escritos debe ser diferente. En el presente trabajo se muestra que los jóvenes universitarios que participaron en el estudio son sensibles al soporte en el que leyeron, los resultados obtenidos en comprensión lectora son dependientes del soporte presentado; por lo que se puede afirmar que la lectura no es independiente del soporte en el que se accede a ella (papel, ordenador, teléfono móvil o celular).

Los ítems presentados fueron capaces de predecir en qué formato obtendrían los participantes mejores resultados en comprensión lectora. Globalmente, el ordenador fue el mejor soporte, seguido por el papel y el teléfono móvil, en contraste con investigaciones previas (Aatonen et al., 2011; Bus y Neuman, 2009) lo que puede estar indicando un cambio en las nuevas generaciones ligado a la familiaridad en el uso de las TIC. Aquellos sujetos que se desempeñan con holgura en las TIC leen mejor en formato digital que los que no están familiarizados.

\section{REFERENCIAS}

Aatonen, M., Mannonen, P., Nieminen, S., y Nieminen, M. (2011). Usability and compatibility of e-book readers in an academic environment: a collaborative study. International Federation of Library Associations, 37(1), 16-27.

Ackerman, R., y Goldsmith, M. (2011). Metacognitive regulation of text learning: On screen versus on paper. Journal of Experimental Psychology: Applied, 17(1), 18.

Bus, A., y Neuman, S.B. (2009). Multimedia literacy development. New York: Routledge.

De Sola, J., Talledo, H., Rubio, G., y Rodríguez, F. (2017). Psychological factors and alcohol use in problematic mobile phone use in the Spanish population. Frontiers in Psychiatry, 8, 126. doi: 10.3389/fpsyt.2017.00011

Jiménez, A., Jiménez, V., Alvarado, J.M., y Puente, A. (2012). A mixed method approach to evaluating reading skills developed by cognitive and metacognitive pedagogic intervention. Valencia: ICERI.

Jiménez, V., Alvarado, J.M., y Llopis, C. (2017). Validación de un cuestionario diseñado para medir frecuencia y amplitud de uso de las TIC. Edutec. Revista Electrónica de Tecnología Educativa, 61, 1-14. doi:10.21556/edutec.2017.61.949 
Jiménez, V., Jiménez, A., Puente, A., y Alvarado, J.M. (2011). Design and evaluation of a metacognition strategies program for students of Secondary Education with difficulties in reading comprehension. ICERI2011 (International Conference of Education, Research and Innovation).

Liu, Z. (2006). Print vs. Electronic resources: A study of user perceptions, preferences, and use.' Information Processing and Management, 42(2), 583-592.

Romero-Otero, I., Iglesias-Fernández, E., y Giménez-Toledo, E. (2013). Use, acceptance and expectations for the ebook in a research library. Bid, 31, 1-10.

Salmerón, L., y García, V. (2012). Children's reading of printed text and hypertext with navigation overviews: The role of comprehension, sustained attention, and visuo-spatial abilities. Journal of Educational Computing Research, 47(1), 33-50.

Sidi, Y., Shpigelman, M., Zalmanov, H., y Ackerman, R. (2017). Understanding metacognitive inferiority on screen by exposing cues for depth of processing. Learning and Instruction, 51,61-73.

Singer, L.M., y Alexander, P.A. (2016). Reading Across Mediums: Effects of Reading Digital and Print Texts on Comprehension and Calibration. Journal of Experimental Education, 85(1), 155-172. doi:10.1080/00220973.2016.1143794

Singer, L.M., y Alexander, P.A. (2017). Reading on paper and digitally: What the past decades of empirical research reveal. Review of Educational Research, 87(6), 1007-1041.

Wang, L., y Lee, K. (2014). The Study of Learners' Preference for Visual Complexity on Small Screens of Mobile Computers Using Neural Networks. Turkish Online Journal of Educational Technology - TOJET, 13(2), 168-180.

Wästlund, E., Reinikka, H., Norlander, T., y Archer, T. (2005). Effects of VDT and paper presentation on consumption and production of information: Psychological and physiological factors. Computers in Human Behavior, 21(2), 377-394.

Yen, C., y Wang, M. (2002). Study of user experiences on electronic- and paper- based reading.

Recibido: 20 de septiembre de 2019

Recepción Modificaciones: 15 de octubre de 2019

Aceptado: 16 de octubre de 2019 